\title{
Rotation Measure variations for millisecond pulsars
}

\author{
Wenming Yan $^{1}$, R. N. Manchester ${ }^{2}$ and Na Wang ${ }^{1}$ \\ ${ }^{1}$ Xinjiang Astronomical Observatory, CAS, 150, Science 1-Street, Urumqi, \\ Xinjiang 830011, China \\ email: yanwm@xao.ac.cn na.wang@xao.ac.cn \\ ${ }^{2}$ CSIRO Astronomy and Space Science, Australia Telescope National Facility, PO Box 76, \\ Epping NSW 1710, Australia \\ email: Dick.Manchester@csiro.au
}

\begin{abstract}
As part of the Parkes Pulsar Timing Array (PPTA) project, frequent observations of 20 millisecond pulsars are made using the Parkes 64-m radio telescope. Variations in the mean position angle of the 20 millisecond pulsars can be studied by the PPTA data being recorded in full-polarization mode. We briefly discuss these results.
\end{abstract}

Keywords. Pulsars: general, ISM: general, radio continuum: stars

\section{Introduction}

Pulsar radiation typically has strong linear polarization. The observed radiation from pulsars can be affected by Faraday rotation occurring in the interstellar medium. Faraday rotation is quantified by the rotation measure $(\mathrm{RM})$, given by

$$
\psi=\mathrm{RM} \lambda^{2},
$$

where $\psi$ is the linear polarization position angle (PA) and $\lambda=c / \nu$ is the radio wavelength corresponding to radio frequency $\nu$. The rotation measure is given by

$$
\mathrm{RM}=0.810 \int_{0}^{D} n_{e} \boldsymbol{B} \cdot d \boldsymbol{l},
$$

where $n_{e}$ is the interstellar electron density in units of $\mathrm{cm}^{-3}, \boldsymbol{B}$ is the vector magnetic field in micro-gauss and the integral is over the path to the pulsar.

Long-term variations in PAs possibly result from changes in the polarization of the emitted radiation or may be due to changes in the RM along the path (see Eq. 1.1). RM changes can occur as the path to the pulsar traverses different regions of the interstellar medium (ISM) or in the Earth's ionosphere due to the diurnal and other changes in the ionospheric total electron content (see Eq. 1.2).

\section{Ionospheric RM corrections}

Because the Earth's magnetic field is relatively strong $(\sim 0.5 \mathrm{G})$ and the electron density in the Earth's ionosphere is relatively high (up to $10^{6} \mathrm{~cm}^{-3}$ ), the Earth's ionosphere makes a significant contribution to the total RM along the path to the pulsar. The contribution of the Earth's ionosphere to the total RM should be estimated and then subtracted so that the measured RM just represents the interstellar contribution. Yan et al. (2011) used two programs to compute the ionospheric RM in the direction of a given source at a given time. After comparison, they found that the one based on the 
International Reference Ionosphere gave the best results. Figure 1 shows an example of the ionospheric correction results using two correction programs.

\section{Results and discussion}

Yan et al. (2011) reported on temporal variations in the mean position angle and implied RM variations of the 20 millisecond pulsars. Figure 2 shows an example of their results. They found that the largest systematic effect was that due to variations in the Earth's ionosphere. There are little or no significant long-term variation in RM after ionospheric correction. The authors point out that the interstellar RM variations are unlikely to be due to localized magnetized regions crossing the line of sight because the implied magnetic fields are too high. Most probably they are statistical fluctuations due to random spatial and temporal variations in the interstellar electron density and magnetic field along the line of sight.

Some other authors (e.g. Weisberg et al. 2004, Han et al. 2006) also found apparent time variations in the interstellar RMs of several pulsars. Earlier authors also found correlated RM and DM variations with time for some other pulsars. Hamilton et al. (1977) and Hamilton et al. (1985) studied the variations of RM and DM for the Vela pulsar. They interpret the RM and DM variations as the result of the relative motion of a magnetized filament of the Vela supernova remnant across the line-of-sight. Van Ommen et al. (1997) made a similar analysis for PSRs B1556-44 and B1727-47. Rankin et al. (1988) made a detailed analysis of the RM and DM variations for the Crab pulsar and found correlated variations between 1972 and 1974 .

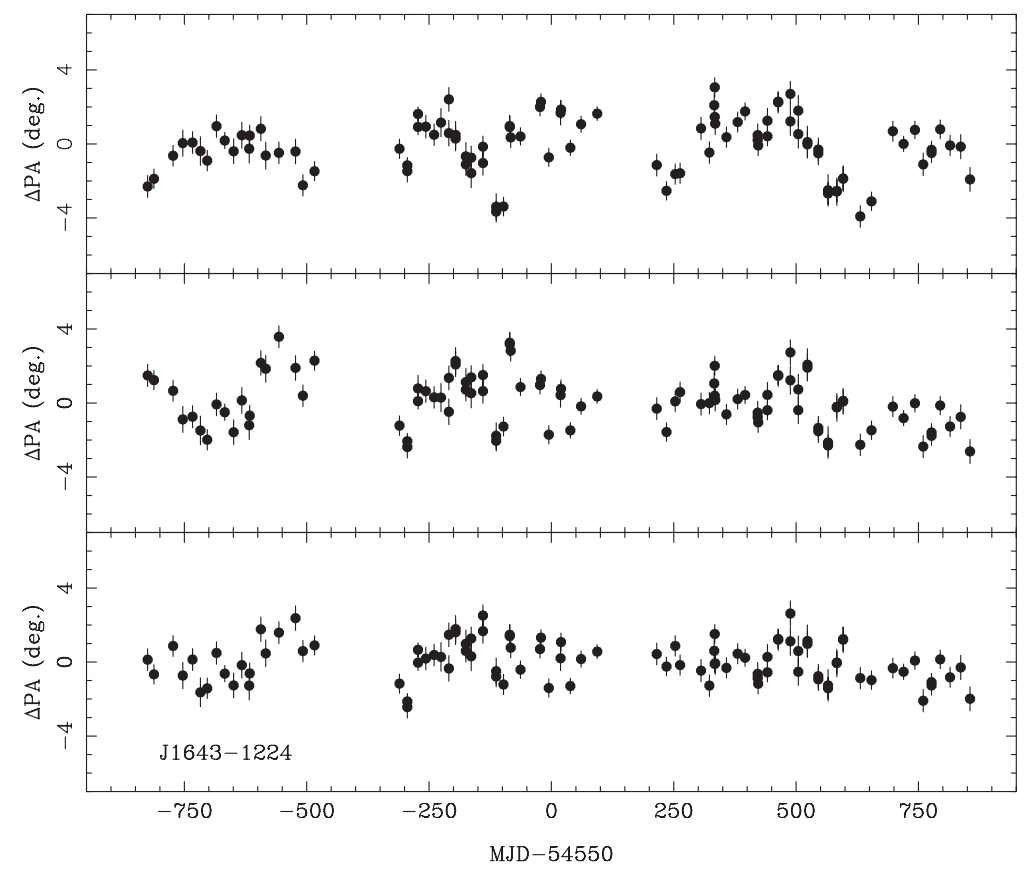

Figure 1. PA differences variations after ionospheric corrections for J1643-1224. The upper panel shows the uncorrected differences and the lower two panels show the corrected PA differences using the two methods, with the IRI-corrected data at the bottom. See Yan et al. (2011) for details. 


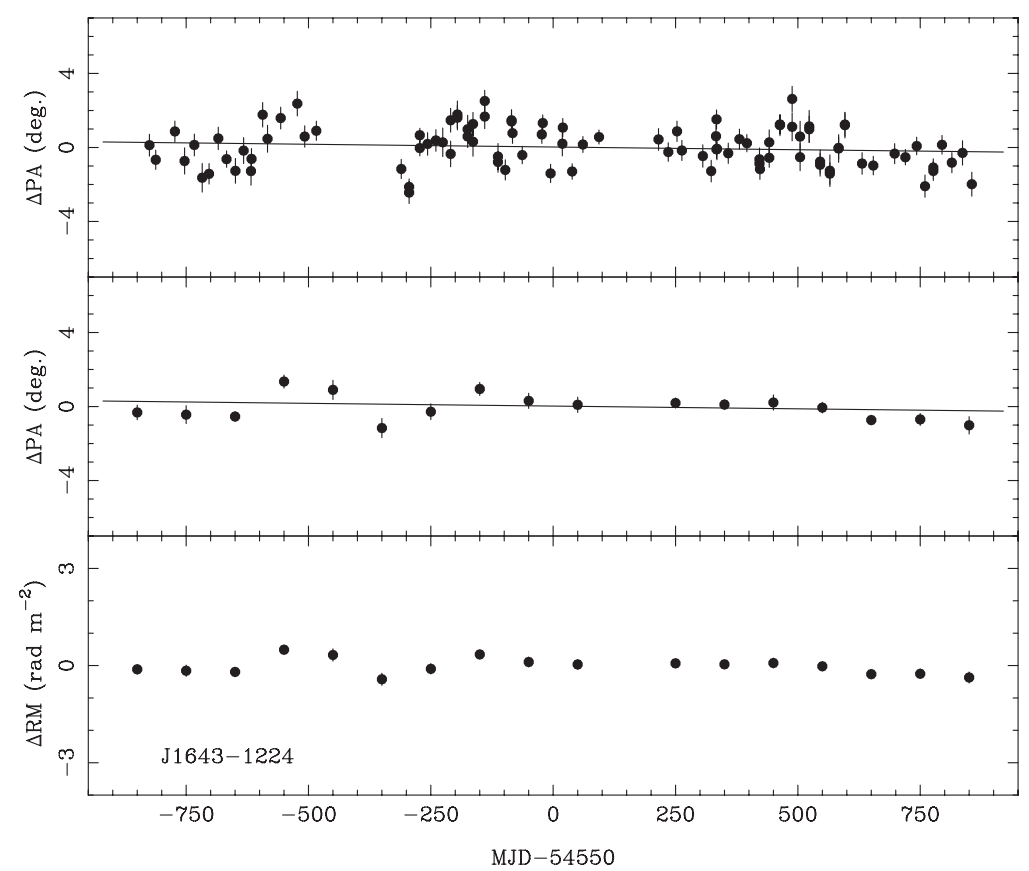

Figure 2. PA and RM variations for PSR for J1643-1224. See Yan et al. (2011) for details.

More recently Yuen et al. (2012) analyzed the changes in the PA of PSR J0737-3039A in the Double Pulsar system around the time of its eclipse by PSR J0737-3039B observed using the Parkes 64-m telescope. They found that differential synchrotron absorption in the closed field-line regions can account for the observed position angle changes during the eclipse. They interpret PA changes after the eclipse as Faraday rotation in the magnetotail of pulsar B. Modeling of the changes in PA after the eclipse with Faraday rotation gives a charge density that is consistent with the Goldreich-Julian value.

\section{Acknowledgments}

WY is supported by NSFC project 11203063 and West Light Foundation of the Chinese Academy of Sciences project XBBS201123.

\section{References}

Hamilton P. A., Hall P. J., \& Costa M. E. 1985, MNRAS, 214, 5

Hamilton P. A., McCulloch P. M., Manchester R. N., Ables J. G., \& Komesaroff M. M. 1977, Nature, 265, 224

Han J. L., Manchester R. N., Lyne A. G., Qiao G. J., \& van Straten W. 2006, ApJ, 642, 868

Rankin J. M., Campbell D. B., Isaacman R. B., \& Payne R. R. 1988, A\& A, 202, 166

van Ommen T. D., D'Alesssandro F. D., Hamilton P. A., \& McCulloch P. M. 1997, MNRAS, 287,307

Weisberg J. M., Cordes J. M., Kuan B., Devine K. E., Green J. T., \& Backer D. C. 2004, ApJS, 150,317

Yan W. M., Manchester R. N., Hobbs G., van Straten W., Reynolds J. E., Wang N., Bailes M., Bhat N. D. R., Burke-Spolaor S., Champion D. J., Chaudhary A., Coles W. A., Hotan A. W., Khoo J., Oslowski S., Sarkissian J. M., \& Yardley D. R. B. 2011, ApE্SSS, 335, 485

Yuen R., Manchester R. N., Burgay M., Camilo F., Kramer M., Melrose D. B., \& Stairs I. H. 2012, ApJ, 752, L32 